\title{
Les îlots verbaux dans les dislocations chez l'enfant français
}

\author{
Morgane Jourdain ${ }^{1,2, *}$ et Emmanuelle Canut ${ }^{2}$ \\ ${ }^{1}$ KU Leuven, Blijde Inkomststraat 21, 3000 Leuven, Belgique \\ ${ }^{2}$ Université Lille 3, Domaine Universitaire du Pont de Bois, 59650 Villeneuve-d'Ascq, France
}

Résumé. Selon les théories basées sur l'usage, au cours de l'acquisition de la syntaxe, l'enfant passe par un stade d'îlot verbal (Tomasello (1992, 2000, 2003). A ce stade, les constructions de l'enfant sont produites avec un verbe précis, et ce verbe n'est pas généralisé à d'autres constructions. Ces études étant basées sur l'acquisition de constructions concernant la structure argumentale des verbes, nous avons voulu tester cette théorie avec un autre type de construction acceptant une grande variation de structures argumentales possibles: la dislocation. Notre étude longitudinale sur les 300 premières dislocations de deux enfants entre 1 et 2 ans met en évidence que, bien que les premières dislocations soient réalisées majoritairement avec un nombre de verbes restreint, ces verbes ne sont pas limités aux dislocations. Cette construction ne semble donc pas passer par un stade d'îlot verbal et semble être le fruit d'un processus d'étayage de schémas « concrets ». Cette différence de processus peut être due au fait que les dislocations concernent la périphérie de l'énoncé, alors que les constructions formant des îlots verbaux semblent concerner la structure interne de l'énoncé.

\begin{abstract}
According to usage-based theories, during syntax acqusition, children's constructions go through a verb-islands stage (Tomasello, 1992, 2000 , 2003). At this stage, children's constructions are produced with a particular verb, and this verb is not generalized to other constructions. These studies being based on the acquisition of constructions concerning the argument structure of verbs, we wanted to test this theory with another type of construction which can be produced with a variety of possible argument structures: dislocation. Our longitudinal study on the 300 first dislocations of two children between age 1 and 2 shows that, even though earliest dislocations are mostly produced with a restricted number of different verbs, these verbs are not limited to dislocation. Therefore, this construction does not seem to go through a verb-island stage and seems to result from a process of extension of more "concrete" schemas. This difference of process can be due to the fact that dislocation deal with the periphery of the utterance whereas constructions going through a verbisland stage concern the internal structure of the utterance.
\end{abstract}

\footnotetext{
*Corresponding author : morgane.jourdain@kuleuven.be
} 


\section{Introduction}

Cet article a pour but d'explorer les théories basées sur l'usage (usage-based) concernant le développement de la syntaxe chez l'enfant, comme les îlots verbaux (verb-islands) ou schémas à pivot (Tomasello, 1992, 2000, 2003) pour une construction n'ayant pas trait à la structure argumentale, la dislocation. Cette construction consiste en la production d'un constituant en périphérie droite ou gauche d'un énoncé, qui est en général repris par un pronom clitique à l'intérieur de l'énoncé. Si l'élément disloqué est omis, le reste de l'énoncé correspond à une structure canonique. La dislocation est donc une construction qui ne concerne pas directement la structure interne de l'énoncé, contrairement à d'autres constructions comme par exemple la construction transitive, causative ou passive qui déterminent quels arguments peuvent être réalisés. Les études de l'approche basée sur l'usage se sont concentrées sur ces constructions concernant l'organisation argumentale et donc sur la structure interne (Lieven et al., 1997). Avec la construction causative, l'enfant doit apprendre qu'un agent et un patient sont nécessaires, alors que les dislocations sont possibles avec tout type d'arguments. Nous allons donc tenter de déterminer si les schémas à pivot ou îlots verbaux peuvent expliquer le développement de tout type de constructions dans le discours de l'enfant, même celles n'influant pas sur la structure argumentale. Pour cela, nous allons étudier la présence de schémas à pivots et des îlots verbaux dans les dislocations de l'enfant. Ces constructions identifiées dans la théorie basée sur l'usage se caractérisent par l'utilisation de lexèmes fixes, rendant leur langage formulaïque. Nous allons donc nous appuyer sur la littérature concernant la théorie basée sur l'usage concernant le développement langagier chez l'enfant et sur la recherche en phraséologie et langage formulaïque.

\subsection{Le langage formulaïque chez l'adulte}

Certaines études de corpus affirment que le langage de l'adulte contient non seulement des mots distincts, mémorisés séparément les uns des autres, mais également certains groupes de mots qui sont utilisés très fréquemment ensemble, de façon plus ou moins fixe (Arnon, 2009; Erman et Warren, 2000; Gonzalez Rey, 2002; Gross, 1996; Sinclair, 1991). Selon Erman et Warren (2000), ces blocs constituent environ la moitié du langage produit par l'adulte. La connaissance de ces blocs constitue même une partie importante de l'acquisition d'une langue puisque dans de nombreux cas, il n'est pas possible de remplacer un mot par un autre de sens identique. Ainsi, Danell (1995) donne l'exemple du bloc jour férié, dans lequel il n'est pas possible de remplacer le mot jour par journée qui a pourtant le même sens : *journée fériée. Un locuteur doit donc non seulement être capable d'associer les mots de la langue en fonction des différentes constructions grammaticales, mais il doit également savoir quels mots peuvent être combinés et quels mots ne sont jamais utilisés ensemble (Pawley et Sydney, 1983). Il a en effet été montré que les locuteurs avaient des connaissances implicites riches sur la fréquence d'apparition de mots en cooccurrence avec d'autres mots (Ellis, 2012; MacDonald et Seidenberg, 2006). L'importance de ces blocs formulaïque dans le développement langagier en acquisition du français L2 est montrée par Perez-Bettan (2015), qui rapporte une proportion plus forte de séquences préfabriquées chez les apprenants plus avancés.

De par leur fréquence, ces blocs permettent d'améliorer la performance du locuteur pour le traitement et la production d'énoncés. Il a en effet été montré que la fréquence et le contexte linguistique de production d'un énoncé jouent un rôle important pour la mémoire, et qu'au plus un élément est fréquent, au plus il devient ancré dans notre mémoire et au plus il est facile à extraire pour être produit (Anderson, 2009; Bybee, 2006; Ellis et al., 2016). Ce type d'expression est donc particulièrement facile à produire dans des énoncés plus larges (Ellis, 2012), et rendent le discours, notamment celui des locuteurs L2, plus 
fluide (Perez-Bettan, 2015). La production de ces blocs est phonétiquement réduite par rapport aux parties du langage ne formant pas de bloc fréquent (Jurafsky et al., 2001). Pour le traitement du langage, une proposition relative objet sera traitée plus rapidement si elle est précédée d'une combinaison sujet-verbe fréquente (Reali et Christiansen, 2007).

La présence de ce type de langage formulaïque se retrouve également dans le discours adressé à l'enfant. Dans une étude de corpus portant sur le discours adressé à l'enfant, Bannard et Matthews (2008) ont montré que certains de ces blocs étaient même plus fréquents que certains lexèmes. Ainsi, les blocs What do you think?, Sit in your chair, ou $a$ cup of tea sont utilisés plus fréquemment que des mots faisant partie du vocabulaire basique comme learn ou pet. Il est donc possible que le langage déjà formulaïque de l'adulte puisse être utilisé par l'enfant dans son apprentissage de la langue.

\subsection{Théories basées sur l'usage de l'acquisition du langage}

De nombreuses études sur l'acquisition de la syntaxe chez l'enfant ont mis en évidence l'existence de constructions contenant du langage formulaïque, en particulier dans le cadre de la théorie basée sur l'usage (Tomasello (1992, 2000, 2003)).

Les théories basées sur l'usage s'opposent aux théories générativistes sur la façon dont le langage se développe chez l'enfant. Ainsi, selon l'hypothèse de continuité (Pinker, 1984), l'enfant possède dès le départ les mêmes catégories syntaxiques que les adultes. C'est-àdire que lorsque l'enfant commence à produire des énoncés, les lexèmes qui les composent appartiennent déjà à différentes catégories syntaxiques, comme les noms ou les verbes. Les premiers énoncés contenant plusieurs lexèmes sont le fruit de constructions abstraites nécessitant l'existence de catégories formelles comme celles du sujet, du groupe verbal ou du déterminant et ne sont pas des répétitions de ce que l'enfant a entendu précédemment (Valian, 1986).

Les travaux de Ninio (1988) vont à l'encontre de l'hypothèse de la continuité. Ninio affirme que l'utilisation des noms ou des verbes est motivée par le système global de la grammaire et par la façon dont les lexèmes sont combinés. Si les règles de combinaison de mots ne sont pas les mêmes chez les enfants et chez les adultes, Ninio conclut que les enfants ne peuvent pas catégoriser les mots de la même façon que les adultes. Tomasello (2003) part du même principe, et essaye de déterminer quelles sont les catégories que l'enfant utilise au cours de son développement langagier. L'un des premiers types d'énoncés de l'enfant est l'holophrase. Un holophrase est une entité linguistique, pouvant être composé d'un lexème ou de plusieurs lexèmes figés, qui fonctionne comme un énoncé complet. Barrett (1982) donne l'exemple de that pour exprimer I want that ou Ball ? pour exprimer Where is the ball? Les holophrases contenant un bloc figé de plusieurs lexèmes sont illustrés dans les exemples (1-3) (Lieven et al., 1992).

(1) Lemme see.

(2) I wanna do it.

(3) Gimme that.

Il est possible que le langage formulaïque du langage de l'adulte adressé à l'enfant (Bannard et Latthews, 2008) soit à l'origine de la présence de ces blocs dans certains des holophrases de l'enfant. A ce stade, l'enfant n'a pas de catégories syntaxiques à proprement parler car il n'y a pas de réelle combinaison de mots à l'intérieur de ces holophrases. Tomasello $(2000,2003)$ a identifié un autre type de construction pouvant contenir un groupe de lexèmes formulaïque dans le langage de l'enfant. Il s'agit de ce qu'il nomme "schéma à pivot», théorie qui se base sur la "grammaire à pivot» de Braine (1976). A ce stade, l'enfant commence à créer ses premières vraies combinaisons d'items linguistiques. Ainsi, une partie du schéma reste figée, mais une autre partie devient variable. 
Tomasello (2000) donne les exemples suivants pour illustrer les schémas à pivot, dans lesquels le $X$ représente l'élément variable :

(4) Where's the X ?

(5) I wanna X.

(6) Put X here.

A ce stade, l'enfant n'a pas encore développé de catégorie syntaxique abstraite telle celle des noms communs. Cependant, tous les lexèmes ne peuvent pas être réalisés dans l'espace variable d'un "schéma à pivot ». L'enfant délimite un groupe de lexèmes, liés sémantiquement les uns aux autres ou non, qui peuvent être utilisés dans cet espace variable (Tomasello, 2003). De plus, l'ordre des éléments d'un schéma à pivot n'influe pas sur le sens de l'énoncé, ce qui montre, selon Tomasello, que l'enfant n'a pas encore de réelle syntaxe à ce stade. Ce type de construction est qualifiée de "concrète " dans la littérature sur la théorie basée sur l'usage (Tomasello, 1992, 2003) car elle est basée sur des lexèmes précis, et les énoncés produits à partir de cette construction ne sont pas basés sur une construction nécessitant des concepts linguistiques abstraits tels que les catégories syntaxiques (noms, verbes, adjectifs, etc.).

Selon Tomasello (2000), les prémices du développement de la syntaxe dans le langage de l'enfant apparaissent dans un type de construction qu'il appelle «îlots verbaux ». Dans ce type de construction, l'enfant semble utiliser des constructions concrètes encore très loin des constructions de l'adulte qui résultent d'une abstraction, mais qui commence à avoir une certaine structure. Tomasello (1992) a mis en lumière le fait que ce type de construction est d'abord réalisé avec un seul verbe, et que ce verbe n'est en général pas réalisé dans d'autres constructions. Au cours du développement langagier, le verbe sera employé petit à petit dans de nouvelles constructions, indépendamment du développement des autres verbes. La grammaire de l'enfant se compose alors d'un inventaire de constructions concrètes, chacune centrée autour d'un seul verbe. S'en suit alors un développement des catégories précurseurs des arguments sémantiques. Il est à ce stade de l'acquisition trop tôt pour parler d'agent ou de patient, mais certaines catégories plus concrètes se développent. Par exemple, pour une construction avec le verbe break, l'enfant développe une catégorie avec tout ce qui peut casser quelque chose, et une autre catégorie concernant les entités pouvant être cassées. Tomasello (1992) a nommé cette théorie "l'hypothèse des îlots verbaux » car chaque verbe fonctionnant indépendamment des autres, chacun dans leur propre système syntaxique, la grammaire de l'enfant à ce stade fonctionne sous formes "d'îles ». D'autres chercheurs ont constaté l'existence de ces îlots verbaux. Lieven et al. (1997) mettent en évidence le fait que 92\% des énoncés de 12 enfants anglophones sont dérivés d'une de leurs 25 constructions concrètes initiales. En plus de ces travaux sur corpus, Tomasello et Brooks (1998) montrent, dans le cadre d'une expérience, que les enfants de moins de 4 ans ont des difficultés à utiliser un nouveau verbe dans une construction transitive qu'ils maîtrisent déjà. Tomasello (2000) explique que c'est uniquement après avoir acquis une plus grande expérience linguistique de l'usage de certaines constructions que les enfants peuvent développer des catégories syntaxiques plus abstraites. Casenhiser et Goldberg (2005); Goldberg et al. (2004, 2005) expliquent également qu'utiliser une construction avec un seul verbe leur permet de généraliser le sens du verbe à l'ensemble de la construction. Selon leurs travaux, les enfants généralisent ainsi le sens de la construction ditransitive grâce au verbe give. Dans ces études, ce verbe est en effet le plus fréquent dans cette construction chez l'enfant, et son sens est généralisé à l'ensemble de la construction.

Certaines études sur l'acquisition des constructions chez l'enfant semblent cependant aller à l'encontre de cette hypothèse. McClure et Pine (2002) montrent que les verbes appris lorsque la longueur moyenne des énoncés de l'enfant atteint 2,0 sont réalisés dans des contextes et avec des arguments plus variés que les verbes appris plus tôt. Cela signifie 
que, même dans le cas de l'existence d'îlots verbaux, tous les nouveaux verbes ne doivent pas passer par ce stade. De plus, les travaux de Childers et Tomasello (2001) et Savage et al. (2002) ont mis en lumière le fait que si un jeune enfant est exposé à un grand nombre de constructions transitives dans un espace de temps restreint, les verbes nouvellement acquis peuvent être utilisés dans des constructions transitives. Il est donc important, face à ces résultats divergents, de documenter en détail les premiers stades de l'acquisition des constructions grammaticales.

Les études sur les îlots verbaux ont été réalisées sur des constructions en lien avec la structure des catégories argumentales telles que celles de l'agent ou du patient. Ainsi, ces études concernent surtout l'acquisition de constructions comme la construction transitive ou intransitive, comme par exemples les études de (Lieven et al., 1997; Savage et al., 2002; Tomasello et Brooks, 1998). L'acquisition par l'enfant de constructions verbales argumentales, i.e. VACs ( V Verb-Argument Constructions ») a été étudiée par Ellis, Römer et O'Donnell (2016) en incluant notamment les effets de la fréquence de mots en cooccurrence mais ces travaux concernent généralement de construction s'appliquant à des constructions ayant une structure argumentale précise. C'est pour cela que nous choisissons de tester l'hypothèse des îlots verbaux sur une construction comme la dislocation, qui peut se construire à partir de constructions argumentales variées.

\subsection{Les dislocations dans le discours de l'enfant}

La dislocation est une construction caractérisée par la réalisation d'un constituant en périphérie gauche (7) ou droite (8) de la phrase (Lambrecht, 1981), et qui est en général repris par un clitique à l'intérieur de la phrase (De Cat, 2002).

(7) Ces romains $s_{i}$, je ne les ${ }_{i}$ aime pas.

(8) Ils I $_{\mathbf{i}}$ sont fous, ces romains $\mathbf{i}_{\mathbf{i}}$.

Il est possible de disloquer le sujet comme dans l'exemple (7), ou l'objet direct (8) ou tout autre fonction syntaxique. Il est également possible de disloquer différentes catégories syntaxiques comme les noms, ou pronoms (Ashby, 1988).

Les études sur l'acquisition des dislocations montrent qu'elles commencent à apparaître à un stade très précoce du développement du langage, dès 1 an et 11 mois (De Cat, 2002). En conséquence, les enfants commencent à produire des dislocations à un stade où leurs constructions sont encore liées à des verbes précis selon les théories basées sur l'usage car c'est vers l'âge de 4 ans que les enfants parviennent à utiliser de nouveaux verbes dans des constructions qu'ils connaissent déjà (Tomasello et Brooks, 1998).

Parisse (2008) a testé l'hypothèse des îlots verbaux avec l'acquisition de la dislocation et les résultats ne montrent pas l'existence d'un lien entre les dislocations produites par les enfants et un verbe précis. Il en conclue que les dislocations des enfants n'étaient pas des îlots verbaux. Cependant, il est important de noter que les enfants étudiés par Parisse (2008) sont tous âgés d'au moins 2 ans, or c'est une construction qui peut apparaître plus tôt chez l'enfant. Il est donc possible que les enfants étudiés soient déjà passés par le stade des îlots verbaux et qu'ils aient atteint le niveau d'une construction plus abstraite avant 2 ans.

\subsection{Questions de recherche}

Le but de notre recherche est de tester l'hypothèse des îlots verbaux sur la dislocation avec des enfants plus jeunes que ceux testés précédemment (Parisse, 2008), afin de vérifier si la théorie basée sur l'usage peut expliquer l'acquisition de tout type de construction ou seulement celle des constructions concernant des catégories sémantiques précises comme 
l'agent, le patient, ou le but par exemple. A partir d'une étude du corpus longitudinal de deux enfants entre 1 et 2 ans, nous cherchons dans cet article à vérifier s'il existe une phase d'îlots verbaux pour les premiers verbes utilisés dans la dislocation.

Nous conduirons ainsi une analyse (i) des verbes produits dans les dislocations et en dehors des dislocations, afin de tester si ces verbes sont effectivement limités à une construction ou s'ils sont utilisés également dans d'autres contextes et (ii) des schémas dits concrets, c'est-à-dire des blocs de lexèmes produits en cooccurrence de façon relativement fréquente, présents dans les dislocations.

\section{Méthodologie}

\subsection{Extraction des données}

\subsubsection{Critères d'extraction}

Lorsqu'un énoncé contenait un élément en périphérie gauche ou droite de la phrase et que cet élément était bien repris à l'intérieur de la phrase par un pronom clitique, nous l'avons interprété et extrait comme étant un cas de dislocation. Cependant, le pronom clitique ou le verbe étant souvent absent dans les premières réalisations de cette construction, il était nécessaire de développer des critères d'extraction supplémentaires. Nous avons choisi d'utiliser les mêmes critères d'extraction que De Cat (2000) : l'ordre des mots, lorsque c'est possible, et l'intonation. Pour l'ordre des mots, Ferdinand $(1993,1996)$ et Labelle et Valois (1996) montrent que les sujets en position postverbale correspondent à des formes précoces de dislocations à droite (9) et selon les analyses de De Cat (2002), les pronoms non-nominatifs situés en position préverbale peuvent être interprétés comme des cas précoces de dislocations à gauche (10).

(9) Range tout seul Grégoire. (Grégoire, 2 ;2.25, (Labelle et Valois, 1996))

(10) Mais moi veux mettre ça. (Anne, 3 ;5.4 (De Cat, 2002))

Lorsqu'un élément correspondant au sujet ou à l'objet de la phrase se trouve en position postverbale mais n'est pas situé juste après le verbe, comme dans l'exemple 9, nous l'avons interprété comme étant un cas de dislocation droite :

(11) Est là, chaperon rouge. (Anaïs, $2 ; 04.20$, corpus Lyon CHILDES)

Dans l'exemple (11), chaperon rouge est nécessairement un élément disloqué à droite. S'il s'agissait d'un attribut du sujet, il aurait dû se situer avant là.

Pour les dislocations dont le clitique est manquant, dans le cas des dislocations gauche, si le seul élément préverbal est un pronom tonique, et dans le cas des dislocations droite, si l'élément disloqué à droite est réalisé directement après le verbe, il n'est pas possible de déterminer s'il s'agit réellement d'une dislocation. Pour ce type d'énoncé, nous avons utilisé un second critère d'extraction qui est l'intonation. Le corpus de Lyon contient les vidéos des interactions adulte-enfant. Ces vidéos ont été converties en fichiers son que nous avons pu analyser avec praat. De Cat (2000) explique de façon succincte que dans le cas des dislocations à gauche, le constituant disloqué reçoit une intonation montante et dans le cas des dislocations à droite, l'élément disloqué est produit après une intonation montante. Afin de déterminer au mieux comment cette intonation montante est réalisée, nous avons analysé l'intonation de plusieurs cas clairs de dislocations, c'est-à-dire des dislocations contenant le pronom clitique, et nous avons ensuite comparé l'intonation des énoncés pour lesquels il y avait une possibilité d'avoir une dislocation à ces cas clairs. Nous avons choisi de ne pas baser notre analyse de l'intonation chez l'enfant à partir des travaux sur l'adulte 
car comme indiqué par De Cat (2000), le contour intonatif des dislocations chez l'enfant n'est pas le même que chez l'adulte. Dans notre corpus, l'élément disloqué à droite (figure 1) ou à gauche (figure 2) dans les cas clairs de dislocation reçoit une intonation montante :

Figure 1. Contour intonatif de l'énoncé C'est à Chloé, ça. (Anaïs, $2 ; 09.16 \mathrm{a}$, corpus Lyon)

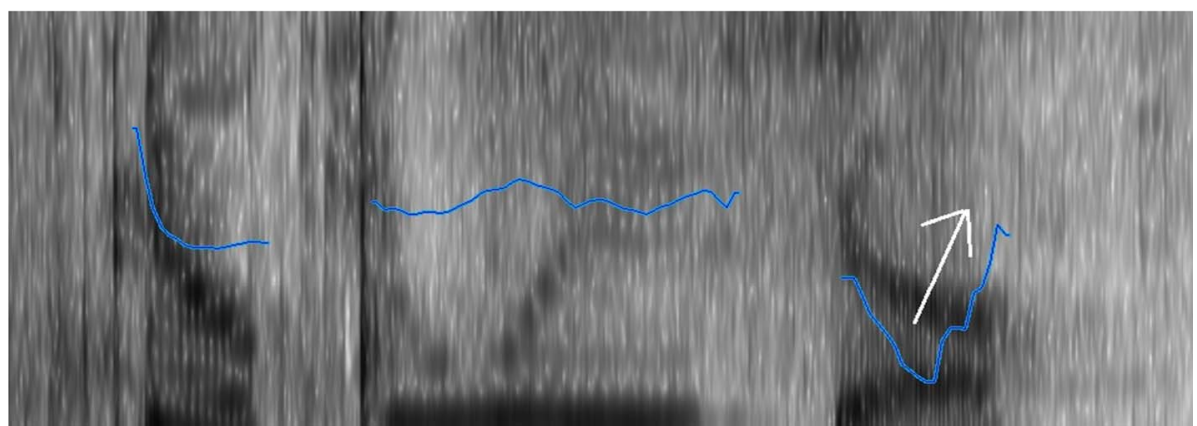

Figure 2. Contour intonatif de l'énoncé Minnie elle est pas cachée. (Anaïs, $2 ; 09.16 \mathrm{a}$, corpus Lyon)

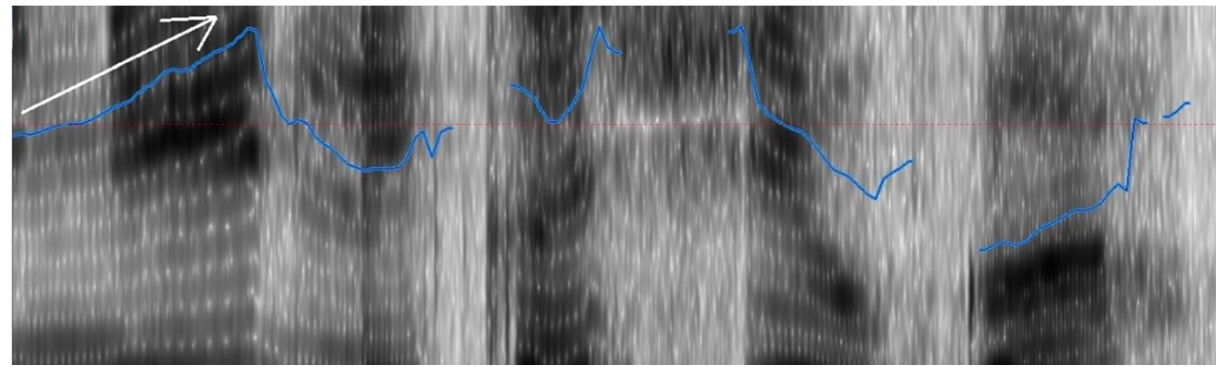

Dans la figure 1, l'énoncé se termine avec une intonation montante sur ça, qui est disloqué à droite, et dans la figure 2, l'énoncé commence avec Minnie qui est disloqué à gauche, repris par le pronom clitique elle et qui reçoit une intonation montante. Nous avons effectué cette analyse sur des enregistrements des mêmes enfants du même corpus, mais collectés lorsque les enfants étaient plus âgées, lorsqu'ils produisaient des énoncés correspondant clairement à des dislocations.

Toutes les dislocations ne présentant pas de clitique ou n'ayant aucun item entre le verbe et l'élément disloqué à droite ont été analysées avec praat, et seules celles avec une intonation montante ont été gardées, comme dans l'exemple en figure 3.

Figure 3. Contour intonatif de l'énoncé Bébé ça. (Anaïs, $2 ; 02.25 \mathrm{a}$, corpus Lyon)

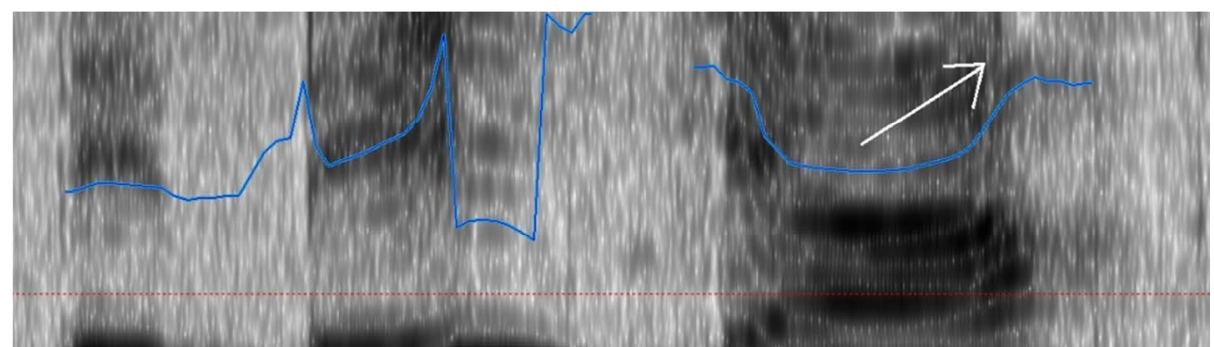




\subsection{2 corpus et sujets}

Nous avons choisi d'analyser les 300 premières dislocations de Marie et d'Anaïs du corpus Lyon (Demuth et Tremblay, 2008). Nous avons également extrait tous les énoncés produits avec un verbe par ces enfants depuis le début des enregistrements jusqu'à celui de leur $300^{\text {ème }}$ dislocation. Ainsi, pour Marie, nous avons extrait tous les énoncés contenant un verbe pour un total de 46 enregistrements, soit 23 heures de dialogue enfant-parent, entre l'âge de $1 ; 0.2$ et 2;2.17, pour arriver à un total de 1346 énoncés. Pour Anaïs, nous avons extrait tous les énoncés contenant un verbe pour un total de 55 enregistrements, soit 27,5 heures de dialogue enfant-parent, entre l'âge de $1 ; 0.23$ et $2 ; 5.11$, pour arriver à un total de 1321 énoncés contenant un verbe.

\subsection{Méthodes d'analyse}

Nous avons testé si les premières dislocations de Marie ou d'Anaïs pouvaient également correspondre aux constructions schématiques telles que décrites par Tomasello (2003). Le langage formulaïque étant très difficile à définir, il n'y a pas de méthodologie parfaite utilisée dans la littérature (Wray, 2002). Nous avons donc simplement basé cette partie de notre analyse sur la fréquence de cooccurrence de deux lexèmes ou plus dont l'un des élément ne peut pas être remplacé, car il s'agit d'un critère, quoiqu'imparfait, raisonnablement fiable et caractéristique de la majorité des blocs de langue formulaïques (Wray, 2002, 2009). Beaucoup d'autres types de critères de figement ont été testés pour le français, mais le blocage lexical, i.e. l'impossibilité de modifier l'un des éléments lexicaux d'un groupe de lexèmes, est le seul à avoir été jugé comme critère nécessaire et suffisant (Svensson, 2004). Pour notre recherche, les lexèmes doivent être utilisés en cooccurrence dans un énoncé, mais il n'est pas nécessaire qu'ils soient produits côte-à-côte, ils peuvent être séparés par un ou plusieurs lexèmes (voir le « schéma à pivot » de l'exemple 6. Ensuite, afin de s'assurer si les verbes présents dans les dislocations chez l'enfant sont uniquement attachés à cette construction ou non, nous avons également fait une analyse de tous les verbes utilisés depuis le début des corpus jusqu'à celui où elle produit sa $300^{\text {ème }}$ dislocation. Nous avons analysé leur fréquence globale dans le discours de l'enfant, ainsi que à le lien avec des constructions particulières ou non.

\section{Résultats}

\subsection{Verbes dans les dislocations}

Sur les 300 premières dislocations de Marie, 105 sont réalisées sans verbe, 109 sont réalisées avec le verbe être, et 86 sont réalisées avec un verbe autre que le verbe être. Les fréquences absolues et relatives de chaque verbe sont données dans le tableau 1.

Tableau 1. Fréquences absolues et relatives des verbes présents dans les dislocations de Marie

\begin{tabular}{|l|l|}
\hline Verbe & Fréquence \\
\hline Verbe absent & $105(35 \%)$ \\
\hline Etre & $109(36,3 \%)$ \\
\hline Vouloir & $28(9,3 \%)$ \\
\hline Mettre & $24(8 \%)$ \\
\hline Faire & $14(4,6 \%)$ \\
\hline Tourner & $3(1 \%)$ \\
\hline Avoir, sauter, tomber, voir & $2(0,6 \%)$ \\
\hline
\end{tabular}




\begin{tabular}{|l|l|}
\hline $\begin{array}{l}\text { Donner, dormir, gagner, manger, monter, } \\
\text { moucher, partir, pédaler, téléphoner }\end{array}$ & $1(0,3 \%)$ \\
\hline Total & $300(100 \%)$ \\
\hline
\end{tabular}

Tableau 2. Fréquences absolues et relatives des verbes présents dans les dislocations d'Anaïs

\begin{tabular}{|l|l|}
\hline Verbe & Fréquence \\
\hline Verbe absent & $188(63 \%)$ \\
\hline Etre & $69(23 \%)$ \\
\hline Tomber & $8(3 \%)$ \\
\hline Arriver & $7(2 \%)$ \\
\hline Aller & $4(1 \%)$ \\
\hline Casser, réparer, trouver & $3(1 \%)$ \\
\hline Vouloir, avoir, écrire, faire, savoir & $2(0,7 \%)$ \\
\hline Boire, essayer, lever, mettre, tenir & $1(0,3 \%)$ \\
\hline Total & $300(100 \%)$ \\
\hline
\end{tabular}

Comme dans l'hypothèse des îlots verbaux, nous avons dans cette construction un verbe qui est très fréquent et qui constitue la majorité des verbes exprimés dans cette construction. Il existe ensuite une liste courte de verbes beaucoup moins fréquents mais tout de même bien présents dans la construction : les verbes vouloir, mettre et faire. Selon l'hypothèse des îlots verbaux, l'existence de ces verbes dans les dislocations de Marie seraient due à un début de la généralisation de la construction.

Une analyse plus précise de ces verbes fréquents révèle l'existence d'une proportion importante de dislocations ayant une structure interne très semblable. Ainsi, parmi les 109 dislocations comprenant le verbe être, 43 sont réalisées sous la forme "Où l'est $+\mathrm{X}$ » ou « $\mathrm{X}+$ où l'est». Il est intéressant de noter que, comme mentionné dans la théorie du schéma à pivot, la place de l'élément variable ne change pas le sens de la phrase:

(12) Où l'est poisson? (Marie18a, $1 ; 11.11$ )

(13) Où l'est ma yaourt ? (Marie20b, $2 ; 0.8$ )

(14) Bougie où l'est? (Marie22b, $2 ; 1.13$ )

15 autres dislocations sont réalisées sous la forme « est là $\mathrm{X}$ » :

(15) Est là banane. (Marie16a, 1;10.11)

(16) Est là biberon de Magalie. (Marie21a, 2;0.28)

Un autre schéma courant est «est à X ça », qui apparaît 8 fois. Dans ce cas, le X fait référence à un possesseur :

(17) Est à Marie ça. (Marie16a, 1;10.11)

(18) C'est à bébé ça. (Marie19b, 1;11.25)

17 dislocations sont produites sous une forme différente que les schémas mentionnés précédemment. Elles prennent la forme « Est PROPRIETE X», dans laquelle PROPRIETE fait référence à un adjectif ou à un nom commun définissant l'élément disloqué. Les premiers exemples de cette construction sont réalisés avec ça à la place de l'élément disloqué (exemples 19 et 20). Dans un second temps, cette partie du schéma devient flexible et d'autres éléments peuvent être réalisés à cette place (exemples 21 et 22):

(19) Est pas bon ca. (Marie16b, 1;10.11)

(20) Est cassé ca. (Marie16b, 1;10.11) 
(21) Est sale le pot. (Marie18b, 1;11.11)

(22) Est très lourd ce bouchon. (Marie22a, 2;1.13)

Pour le verbe mettre, il est possible d'identifier le schéma " met là X » qui apparaît 12 fois pour un total de 22 dislocations avec ce verbe, correspondant à plus de la moitié des cas. Pour 3 cas, le X correspond à l'agent (23), pour 9 cas il correspond au patient (24) :

(23) Marie elle met là Marie. (Marie18a, 1 ;11.11)

(24) Met là ça. (Marie19b, $1 ; 11.25$ )

Dans un deuxième temps, il est possible que ce schéma devienne plus abstrait pour atteindre la forme «met BUT X». Comme il n'y a que 2 cas parmi les dislocations étudiées, nous ne pouvons pas affirmer avec certitude qu'il s'agit là d'une évolution du schéma mentionné précédemment :

(25) Mettre à Marie ça. (Marie20a, 2;0.8)

(26) Je mets la bouche le médicament. (Marie22a, 2;1.13)

Cela signifie que $76 \%$ des dislocations réalisées par Marie avec le verbe être appartiennent à un schéma concret. Pour Anaïs, $87 \%$ des dislocations avec le verbe être contiennent également un schéma. Le schéma le plus fréquent est " est à X ça », et apparaît 21 fois. Ce schéma a la même forme que celui de Marie en (17) et (18) :

(27) C'est à moi ca. (Anaïs22b, $2 ; 0.25$ )

(28)C'est à Chloé ça ? (Anaïs27b, 2 ;5.23)

Le deuxième schéma similaire à celui de Marie est celui sous la forme « est là » (voir (15) et (16) pour la comparaison avec Marie). Ce schéma apparaît 12 fois :

(29) Est là le vélo. (Anaïs22a, $2 ; 0.25$ )

Le troisième schéma d'Anaïs a la forme « est où » et apparaît 14 fois

(30) Est où le violet ? (Anaïs27b, $2 ; 5.23$ )

Enfin, le dernier schéma d'Anaïs a la forme «est X ça » et apparaît 13 fois. Le X est un élément libre dans lequel est inséré un nom commun ou un pronom servant à identifier une entité :

(31) Est le feuç. (Anaïs27a, $2 ; 5.23$ )

(32) Est un canard ça. (Anaïs $27 b, 2 ; 5.23$ )

Cela signifie que la grande majorité des premières dislocations des enfants de notre étude sont réalisées avec un groupe de lexèmes précis, avec un seul lexème o de l'énoncé pouvant changer en fonction de la situation à décrire. Cependant, il ne s'agit pas d'un seul schéma pour une construction, comme prédit par l'hypothèse des îlots verbaux. De plus, l'élément libre dans ces schémas n'est pas toujours au même endroit. Parfois, c'est l'élément disloqué qui est libre (exemples 12-16) ou un élément à l'intérieur de la phrase (exemples 17 et 18). Pour le verbe mettre, un schéma a pu être identifié, et il correspond à plus de la moitié des dislocations avec le verbe mettre. Nos résultats semblent donc indiquer l'existence de constructions concrètes basées sur des mots précis, mais le même verbe sous la même forme apparaît dans différentes constructions. Le terme d' "îlot» ne semble donc pas correspondre exactement à nos données, car soit plusieurs schémas existent pour un même verbe (verbe être), soit le schéma ne peut pas expliquer tous les cas (verbe mettre).

Il est important de noter également la forte proportion de dislocations sans verbes $(35 \%$ des dislocations de Marie et $63 \%$ des dislocations d'Anaïs). Ces énoncés sont 
majoritairement composés d'un prédicat dont le verbe être serait omis et qui est suivi d'un élément disloqué à droite :

(33) Méchant ça. (Anaïs25a, $2 ; 2.25$ )

(34) A moi ceux-là. (Anaïs $25 b, 2 ; 2.25$ )

(35) Pas là ça. (Anaïs27b, 2 ;5.23)

(36) Soleil ça (Anaïs30b, $2 ; 5.11$ )

Les recherches précédentes affirment que les enfants développent leurs constructions à partir des verbes qu'elles contiennent (Casenhiser et Goldberg, 2005; Goldberg et al., 2004, 2005). Le verbe est souvent décrit comme étant le moteur de l'acquisition des constructions syntaxiques (Tomasello, 1992, 2000, 2003; Tomasello et Brooks, 1998). Le fort taux de dislocations ne contenant pas de verbe semble donc suggérer que les constructions sans lien avec la structure argumentale ne dépendent pas d'un verbe pour se développer, et que l'enfant peut utiliser de telles constructions sans verbe au départ.

\subsection{Verbes hors dislocations}

Durant la période étudiée, Marie a produit 1364 énoncés avec un verbe. Parmi ces 1364 énoncés, 346 contenaient le verbe être, dislocations comprises, soit $25 \%$ du nombre total d'énoncés avec un verbe. Ce chiffre est inférieur au pourcentage du nombre d'occurrences du verbe être dans les dislocations, mais il est tout de même élevé. De plus, cela signifie que seulement $30 \%$ des occurrences du verbe être concernent des dislocations. Il devient donc difficile d'affirmer que le verbe être soit particulièrement attaché aux dislocations par rapport aux autres constructions. Le même problème se pose pour les autres verbes fréquents dans les dislocations de Marie. Vouloir apparait 80 fois dans son corpus, dont 28 fois dans des dislocations, soit seulement 35\% des cas, et mettre apparaît 155 fois en tout mais seulement 24 fois dans des dislocations, soit seulement $15 \%$ des cas. L'hypothèse des îlots verbaux semble donc ne pas expliquer le cas des dislocations, car les verbes utilisés dans cette construction sont majoritairement utilisés dans d'autres contextes, en dehors de cette construction. La proportion des verbes fréquents dans et en-dehors des dislocations de Marie est indiquée dans le tableau 3.

Tableau 3. Fréquences absolues et relatives des verbes présents dans et hors des dislocations de Marie

\begin{tabular}{|c|c|c|c|}
\hline Verbe & $\begin{array}{l}\text { Fréquence dans les } \\
\text { dislocations }\end{array}$ & $\begin{array}{lr}\text { Fréquence } & \text { en } \\
\text { dehors } & \text { des } \\
\text { dislocations } & \\
\end{array}$ & Fréquence total \\
\hline$\hat{E}$ tre & $109(30 \%)$ & $255(70 \%)$ & $364(100 \%)$ \\
\hline Mettre & $24(15 \%)$ & $131(85 \%)$ & $155(100 \%)$ \\
\hline Vouloir & $28(35 \%)$ & $52(65 \%)$ & $80(100 \%)$ \\
\hline Faire & $14(32 \%)$ & $30(68 \%)$ & $44(100 \%)$ \\
\hline
\end{tabular}

Toutefois, une analyse des schémas présents dans les énoncés ne contenant pas de dislocations met en lumière l'existence d'une grande similarité entre le contenu des dislocations de Marie et celui de ses autres énoncés. Ainsi, le schéma " où l'est », version simplifiée du schéma « Où l'est X », utilisé seul, sans autre élément, est utilisé 10 fois par Marie, et le schéma «est là », version courte du schéma " est là X », est utilisé 51 fois en isolation. Pour le schéma «met là X », la version sans dislocation « met là » est utilisée 63 fois dans le corpus de Marie. Il peut être utilisé de façon isolée ou avec un clitique sujet :

(37) On met là. (Marie19b, 1;11.25) 
(38) Je mets là (Marie21a, 2;0.28)

Le schéma réduit " met là » est donc utilisé dans $48 \%$ des énoncés avec le verbe mettre si on inclut les dislocations. Cela signifie que les schémas identifiés dans les dislocations de Marie ne sont pas en réalité des schémas propres aux dislocations. L'élément disloqué semble venir se greffer sur des schémas existants et relativement fréquents dans le langage de l'enfant. Les dislocations ne forment donc pas des îlots verbaux en elles-mêmes et l'hypothèse des îlots verbaux ne semblent pas non plus correspondre tout à fait aux schémas de nos données puisque pour le verbe être par exemple, plusieurs schémas concrets ont été trouvés.

\subsection{Discussion}

Notre corpus contient des schémas, correspondant aux schémas décrits dans la littérature sur la théorie basée sur l'usage (Bowerman, 1976; Braine, 1976; Tomasello, 2000, 2003). La majorité des dislocations est réalisée avec un nombre restreint de verbes. Dans notre corpus, seuls 4 verbes peuvent être qualifiés de fréquents dans les dislocations de Marie et 3 verbes pour les dislocations d'Anaïs. Chacun de ces verbes est utilisé dans un ou plusieurs schémas très concrets, dans lesquels le verbe est associé à d'autres mots précis. Le verbe mettre est ainsi majoritairement associé au mot là, et le verbe être est fréquemment utilisé avec où. Seulement, contrairement à l'hypothèse des îlots verbaux de Tomasello (1992, 2000, 2003), un même verbe peut être utilisé dans plusieurs schémas concrets, le verbe être étant utilisé dans pas moins de 4 schémas. Les schémas que nous avons identifiés ne se mélangent pas entre eux et donc ils forment bien des îlots séparés les uns des autres. Toutefois, un verbe précis n'est pas forcément utilisé sur un seul de ces îlots, mais il peut l'être dans un groupe restreint d'îlots.

Un autre point de divergence concerne le développement d'une construction à partir d'un schéma. Bien qu'il semble que l'enfant de notre étude se base sur des schémas précis pour développer la dislocation, il ne semble pas que ces schémas soient propres à cette construction. Certains de ces schémas sont parfois plus souvent utilisés sans dislocation qu'avec. C'est le cas du schéma «met là », qui n'est réalisé sous la forme de dislocation que dans $16 \%$ des occurrences de ce schéma. De plus, nous avons pu identifier pas moins de 5 schémas dans lesquels la dislocation apparaît pour Marie, et 3 schémas pour Anaïs. La dislocation ne semble donc pas se développer à partir de son propre schéma mais de se rattacher à des schémas déjà existants. Tomasello (2000) a en effet expliqué que la tâche de l'enfant lors du développement de ses premières constructions concrètes en constructions abstraites était double : séparer les mots composants leurs blocs de lexèmes figés, et étayer leurs constructions. La dislocation semble donc être une stratégie d'étayage, contrairement au développement de VACs à partir des îlots verbaux. L'enfant part de schémas qu'il maîtrise, leur formulaïcité facilitant sûrement leur production, et qu'il utilise fréquemment et rajoute l'élément disloqué en périphérie droite ou gauche. La dislocation permet d'étayer facilement les schémas de l'enfant, puisque c'est une construction qui concerne surtout la périphérie de la phrase. Il est possible que la stratégie de l'enfant pour développer une construction, les îlots verbaux ou l'étayage, dépende du type de construction en cours d'acquisition. Ainsi, les recherches sur les dislocations ont montré que tout type d'élément à l'intérieur d'une phrase pouvait être disloqué (Ashby, 1988; Barnes, 1985; Blasco, 1997; De Cat, 2002). La dislocation est donc compatible avec un grand nombre d'autres constructions, la structure interne de la phrase peut contenir une variété de structures argumentales. La construction transitive, qui est la construction la plus étudiée dans le cadre de la recherche sur les îlots verbaux (Childers et Tomasello, 2001; Lieven et al., 1997), porte vraiment sur la structure argumentale du verbe et donc la structure interne de 
la phrase. Ces constructions sont donc fondamentalement différentes et pourraient expliquer cette différence de stratégie d'abstraction de constructions concrètes.

Un autre élément de nos données diffère des analyses précédentes des théories basées sur l'usage. Il a été montré que le verbe était l'un des éléments centraux dans l'acquisition d'une VAC (Goldberg et al., 2004, 2005). Néanmoins, les enfants de notre corpus produisent des dislocations bien avant de systématiquement produire le verbe dans cette construction, puisque la vaste majorité des premières réalisations de cette construction n'ont pas de verbe. Il est donc intéressant de tester si ces énoncés sans verbes font usage de la dislocation avec la fonction qu'elle est censée avoir dans le langage de l'adulte. La dislocation n'a pas de "sens" sémantique. Le rôle de cette construction se trouve au niveau de la structure de l'information, puisqu'elle sert à exprimer le topique de la phrase (Lambrecht, 1981, De Cat 2002, 2007). Le topique correspond à ce sur quoi porte le discours, et le reste de l'énoncé correspond au commentaire, qui est une information donnée au sujet du topique (Lambrecht, 1994). Typiquement, l'élément réalisé à la position du topique est une entité mentionnée précédemment dans le discours, tandis que le commentaire correspond à un élément nouveau dans le discours (Lambrecht, 1994). Les recherches antérieures sur l'acquisition de la dislocation ont montré que les éléments disloqués de l'enfant pouvaient être interprétés comme topiques, comme dans le langage de l'adulte (De Cat 2002, 2007). De plus, les dislocations sans verbe du corpus que nous avons analysées sont composées d'un élément prédicatif et de l'élément disloqué correspondant au topique, ce qui semble correspondre aux prémices de la relation topiccommentaire que l'on trouve dans les dislocations chez l'adulte. Par conséquent, l'enfant semble être capable d'utiliser la dislocation de façon appropriée quant à sa fonction, avant même d'avoir commencé à l'utiliser de façon systématique avec un verbe. Contrairement aux VACs, l'enfant n'a donc pas extrait le «sens » de la construction à partir d'un verbe très fréquent. Il est également possible que les dislocations se développent différemment des VACs car elles n'ont pas réellement un sens dans la définition sémantique du terme, mais plutôt une fonction pragmatique au niveau de la structure de l'information. Il serait intéressant d'étudier le développement d'autres constructions à fonction pragmatique plutôt que sémantique afin de déterminer si le verbe a effectivement une importance moindre dans certains types de constructions que dans d'autres.

Une étude comparative de l'acquisition de constructions à structure argumentale et d'autres types de construction pour un même enfant permettrait d'étudier plus finement la différence de processus lors du passage d'un schéma concret à une construction abstraite, c'est-à-dire une construction que l'enfant a schématisé avec des notions linguistiques abstraites comme les catégories syntaxiques lui permettant de construire des exemplaires de la construction avec les lexèmes de son choix au lieu de devoir se limiter à un lexème obligatoire pour la construction, avec une comparaison entre des constructions ayant une structure argumentale et celles qui n'en ont pas.

\section{Conclusion}

Dans cet article, nous avons essayé de déterminer si l'enfant passait par un stade d'îlot verbal dans l'acquisition de la dislocation. Nous avons analysé les verbes présents dans les dislocations et dans les autres constructions produites par des enfants entre $1 ; 0.2$ et $2 ; 5.11$, afin de déterminer si les verbes utilisés dans les dislocations sont bien propres à cette construction comme prédit par l'hypothèse des îlots verbaux.

Le premier résultat est que les enfants de notre étude passent par un stade où les constructions sont produites avec certains lexèmes précis, qui semblent former des blocs entre eux. Les premières dislocations semblent fonctionner autour de différents blocs. Ainsi, il n'y a pas un schéma utilisé pour les dislocations, mais plusieurs. 
Le deuxième résultat est que les schémas et verbes fréquemment utilisés dans les dislocations ne sont en réalité pas propres aux dislocations. Les verbes les plus fréquents dans cette construction sont également les verbes les plus fréquents en général dans le discours de l'enfant. De plus, ces verbes fréquents semblent utilisés dans des schémas précis qui se sont formés avant l'apparition des dislocations dans le discours de l'enfant. La dislocation n'émerge pas de son propre schéma concret, mais apparaît sur des schémas déjà formés pour d'autres constructions.

Le troisième et dernier résultat concerne l'absence de verbes dans les premières dislocations, alors que la fonction de la structure de l'information de la construction est déjà en place. Contrairement aux travaux basés sur l'usage réalisés sur des «VACs", le sens de la construction n'est pas généralisé à partir du sens du verbe le plus fréquent puisque dans le cas de notre étude, le sens est en place avant que le verbe ne soit majoritairement réalisé dans la construction. Ce résultat suggère que les constructions autres que VACs passent par un processus d'acquisition différent.

Selon Tomasello (2000), à partir du stade des holophrases, l'enfant a une tâche double : séparer les mots qui composent l'holophrase et étayer cette construction. La théorie des îlots verbaux semble découler de la première tâche, mais la deuxième a été moins développée dans les théories basées sur l'usage. La façon dont la dislocation se met en place dans le langage de l'enfant semble illustrer une façon dont l'enfant étaye ses constructions : il part d'un schéma qu'il maîtrise et qu'il a l'habitude d'utiliser, et ensuite il rajoute des éléments avant ou après le schéma. Cette différence de stratégie est peut-être due à la différence dans le type de construction étudié dans les précédentes recherches sur les îlots verbaux et celui étudié dans le présent article. Les constructions étudiées à travers la théorie des îlots verbaux concernent le noyau de la phrase, puisqu'elles déterminent la structure des arguments du verbe. Par contre, les dislocations concernent la périphérie de l'énoncé. Cependant, d'autres recherches sur ces deux types de constructions sont nécessaires afin de vérifier l'existence de ces deux méthodes de développement syntaxique chez l'enfant.

\section{Références}

Anderson, J. R. (2009). Cognitive psychology and its implication. New York: Worth.

Arnon, I. (2009). Starting big - the role of multi-word phrases in language learning and use. (Doctor of Philosophy), Stanford University.

Ashby, W. (1988). The syntax, pragmatics and sociolinguistics of left- and right-dislocations in French. Lingua, 75, 203-229.

Bannard, C., \& Matthews, D. (2008). Stored word sequences in language learning. Psychological science, 19(3), 241-248.

Barnes, B. K. (1985). Left Detachment in Spoken Standard French (Vol. VI:3). Amsterdam: Benjamins Publishing Company.

Barrett, M. (1982). The holophrastic hypothesis: conceptual and empirical issues. Cognition, 11, 4776.

Blasco, M. (1997). Pour une approche syntaxique des dislocations. French Language Studies, 7, 1-21.

Bowerman, M. (1976). Semantic factors in the acquisition of rules for word use and sentence construction. In D. Morehead \& A. Morehead (Eds.), Norman and Deficient Child Language. Baltimore: University Park Press.

Braine, M. (1976). Children's first word combinations (Vol. 41). Chicago: Chicago University Press. Bybee, J. (2006). From usage to grammar: the mind's response to repetition. Language, 82, 711-733.

Casenhiser, D., \& Goldberg, A. (2005). Fast mapping between a phrasal form and meaning. Developmental Science, 8, 500-508. 
Childers, J., \& Tomasello, M. (2001). The role of pronouns in young children's acquisition of the English transitive construction. Development psychology, 37, 730-748.

Danell, K. J. (1995). Le phénomène de concurrence en français moderne : réflexions à partir de anannée, jour-journée, paraître-apparaittre, et d'autres. Uppsala: Swedish Science Press.

De Cat, C. (2000). Structure Building and the Acquisition of Dislocations in Child French. Paper presented at the Annual Boston University Conference on Language Development, Boston.

De Cat, C. (2002). French Dislocation. (Doctor of Philosophy), University of York.

Demuth, K., \& Tremblay, A. (2008). Prosodically-conditioned variability in children's production of French determiners. Journal of Child Language, 35(1), 99-127.

Ellis, N. C. (2012). Formulaic language and second language acquisition: Zipf and the phrasal teddy bear. Annual Review of Applied Linguistics, 32, 17-44.

Ellis, N. C., Römer, U., \& O'Donnell, M. B. (2016). Language usage, acquisition, and processing: Cognitive and corpus investigations of construction grammar. Malden, MA: Wiley-Blackwell.

Erman, B., \& Warren, B. (2000). The idiom principle and the open choice principle. Text, 20(1), 2962.

Ferdinand, A. (1993). Subject dislocations in French child language. HIL Manuscripts.

Ferdinand, A. (1996). The development of functional categories - the acquisition of the subject in French (Vol. 23). The Hague: Holland Academic Graphics.

Goldberg, A., Casenhiser, D., \& Sethuraman, N. (2004). Learning argument structure generalisations. Cognitive Linguistics, 15, 289-316.

Goldberg, A., Casenhiser, D., \& Sethuraman, N. (2005). Role of prediction in construction learning. Journal of Child Language, 32, 407-426.

Gonzalez Rey, I. (2002). La phraséologie du français. Toulouse: Presses Universitaires du Mirail.

Gross, G. (1996). Les expressions figées en français; noms composés et autres locutions. Paris: Éditions Ophrys.

Jurafsky, D., Bell, A., Gregory, M., \& Raymond, W. (2001). Probabilistic relations between words: Evidence from reduction in lexical production. In J. Bybee \& P. Hopper (Eds.), Frequency and the emergence of linguistic structure (pp. 229-254). Amsterdam: John Benjamins.

Labelle, M., \& Valois, D. (1996). The status of post-verbal subjects in French child language. Probus, $8(1), 53-80$.

Lambrecht, K. (1981). Topic, Antitopic and Verb Agreement in Non-Standard French (H. a. V. Perret, Jef Ed. Vol. II:6.). Amsterdam: John Benjamins B.V.

Lieven, E., Pine, J. M., \& Baldwin, G. (1997). Lexically-based learning and early grammatical development. Journal of Child Language, 24, 187-220.

Lieven, E., Pine, J. M., \& Dresner Barnes, H. (1992). Individual differences in early vocabulary development. Journal of Child Language, 19, 287-310.

MacDonald, M. C., \& Seidenberg, M. S. (2006). Constraint satisfaction accounts of lexical and sentence comprehension. In M. J. Traxler \& M. A. Gernsbacher (Eds.), Handbook of psycholinguistics (pp. 581-611). London: Elsevier Inc.

McClure, K., \& Pine, J. M. (2002). Examining the verb island hypothesis. Paper presented at the Ninth International Congress for the Study of Child Language and the Symposium on Research in Child Language Disorders, Madison.

Ninio, A. (1988). On formal grammatical categories in early child language In Y. Levy, I. M. Schlesinger, \& M. D. S. Braine (Eds.), Categories and processes in language acquisition. Hillsdale, NJ: Lawrence Erlbaum Associates.

Parisse, C. (2008). Left-dislocated subjects: A construction typical of young French-speaking children? In J. Benjamins (Ed.), First Language Acquisition of Morphology and Syntax: Perspectives across Languages and Learners (pp. pp.13-30).

Pawley, A., \& Sydney, F. H. (1983). Two puzzles for linguistic theory: nativelike selection and nativelike fluency. In J. J. C. Richards \& R. R. W. Schmidt (Eds.), Language and communication (pp. 191-225). London: Longman.

Perez-Bettan, A. (2015). Apprentissage et utilisation du langage préfabriqué chez des apprenants de français langue étrangère. $(\mathrm{PhD})$, University of Paris 8 , Paris.

Pinker, S. (1984). Language learnability and language development (Vol. 7). Cambridge, Massachusetts and London, England: Harvard University Press.

Reali, F., \& Christiansen, M. H. (2007). Processing relative clauses is made easier by frequency of occurrence. Journal of Memory and Language, 57, 1-23. 
Savage, C., Lieven, E., Theakston, A., \& Tomasello, M. (2002). Priming transitive constructions with 3-year-olds, 4-year-olds and 6- to 7-year-olds. Paper presented at the Ninth International Congress for the Study of Child Language and the Symposium on Research in Child Language Disorders, Madison.

Sinclair, J. (1991). Corpus, concordance, collocation. Oxford: Oxford University Press.

Svensson, M. H. (2004). Critères de figement, l'identification des expressions figées en français contemporain. $(\mathrm{PhD})$, Umeå Universitet

Tomasello, M. (1992). First verbs: A case study of early grammatical development: Cambridge University Press.

Tomasello, M. (2000). First steps toward a usage-based theory of language acquisition. Cognitive Linguistics, 11(1), 61-82. doi:0936-5907/00/0011-0061

Tomasello, M. (2003). Constructing a Language, A Usage-Based Theory of Language Acquisition (H. U. Press Ed.): Cambridge, Massachusetts and London, England.

Tomasello, M., \& Brooks, P. (1998). Young children's earliest transitive and intransitive constructions. Cognitive Linguistics, 9, 379-395.

Valian, V. (1986). Syntactic categories in the speech of young children. Development psychology, 22, $562-579$. 